\title{
Grain Growth Behaviors Observed by a Modified Potts-MC-type Simulation
}

\author{
Kunio ITO* \\ Department of Materials Engineering, The University of Tokyo, Bunkyo-ku, Tokyo, 113-8656 Japan. \\ (Received on February 28, 2017; accepted on April 26, 2017)
}

\begin{abstract}
Details of the Potts-MC-type simulation model modified to reduce computing time were explained and effects of parameters to control anti-freezing of the structure, orientation-relation-dependent migration rate and energy of boundaries as well as orientation-relation-dependent migration rate of triple lines on the elementary structural development process were presented. Some topological characters of the structures observed by the modified method were then compared with earlier reported ones. Most of them coincided with the earlier reported results. The critical grain size has been found to coincide with the topological mean grain size. The transition probabilities of grains between topological classes in the shortest elapsed time are reported for the first time; they suggest that the steady state topological grain size distribution could exist for only a short period.
\end{abstract}

KEY WORDS: boundary mobility; boundary energy; grain growth; number of faces of a grain; simulation; topological class; triple line mobility.

\section{Introduction}

Crystallographic textures of metallic materials change by an annealing process through grain growth as well. ${ }^{1-3)}$ Computer simulations are expected to yield important information about factors controlling the grain growth process. In the present paper, details of a simulation model which can handle orientation-relation-dependent grain boundary energy and mobility as well as the triple line mobility were first explained, effects of parameters on boundary energy and mobility as well as triple line mobility were namely demonstrated. Some topological characters of the structures observed for the fundamental grain coarsening process by the present method were then compared with earlier reported ones and the validity of the method was checked. The used simulation algorithm ${ }^{4}$ ) was developed from the two-dimensional algorithm, ${ }^{5)}$ where time needed to compute was remarkably short and it belongs to varieties of the Potts-MC model. ${ }^{6-8)}$

Application of this modelling to a method of hybridization of simulation and statistics to study grain growth texture development shall be given in the following report.

\section{Modelling}

A specimen was created with $256 \times 320 \times 256$ cubic cells with periodic boundary conditions. The orientation of a grain was designated by an orientation number, which represents one of serial numbers of points in the orientation space. The cells constructing a grain share the same

\footnotetext{
* Corresponding author: E-mail: ito_e-prof@material.t.u-tokyo.ac.jp
} DOI: http://dx.doi.org/10.2355/isijinternational.ISIJINT-2017-110 orientation number. A crystallographic texture of grains in a specimen was composed of two components: matrix component $\mathrm{M}$ and background component B. Grains were assigned through a table to one of the texture components.

The energy of cell $i$, the origin of the boundary as well as the triple line energy, was given by Eq. (1);

$$
E\left(O_{i}\right)_{i}=\left(\sum_{n=1}^{26} f_{\text {in }} \overline{\delta_{\text {in }}}\right) / 100
$$

where $n$ represents the serial number of cells in the Moore neighborhood of cell $i$, and $O_{i}$ and $O_{n}$ are the current orientation number of cells $i$ and $n$, respectively. $\overline{\delta_{i n}}$ is equal to 1 for $O_{i} \neq O_{n}$ and 0 for $O_{i}=O_{n}$. When the combination of texture components corresponding to $O_{i}$ and $O_{n}$ belongs to one of the higher boundary energy combinations, $f_{\text {in }}$ is equal to 100 , otherwise $f_{\text {in }}$ is equal to $F(\leq 100)$. Boundary energy factor $F$ controls, by the same rule, the orientation relation dependence of the boundary as well as the triple line energy. $E\left(O_{i}\right)_{i}$ and $F$ were handled as integer in the computer.

When cell $i$ was occupied by cell $j$, or when the orientation number of cell $i$ was altered from $O_{i}^{\text {before }}$ to $O_{i}^{\text {after }}\left(=O_{j}\right)$, we calculated the energy change by Eq. (2);

$$
\Delta E=E_{i}\left(O_{i}^{\text {after }}\right)-E_{i}\left(O_{i}^{\text {before }}\right) .
$$

The occupation was permitted by probability $p$;

$$
p=p(\Delta E) / R_{\text {permission }}
$$

$p(\Delta E)=1$ for $\Delta E \leq 0$ and $p(\Delta E)=\exp (-\Delta E / k T)$ for $\Delta E>0$

where $k T$ is a parameter to avoid freezing of the structure. The multiplicity of occupation event $m$ was calculated as the number of grains to which the cells in the von Neumann neighborhood of cell $i$ belonged. Boundary migration and 
triple line migration correspond to $m=2$ and $m=3$, respectively.

When, in the case of boundary migration, the combination of two texture components corresponding to $O_{i}^{\text {before }}$ and $O_{j}$ belongs to one of the higher boundary mobility combinations, $R_{\text {permission }}$ is equal to 1 , otherwise $R_{\text {permission }}$ is equal to $R(\geq 1.0)$. Similarly, in the case of triple line migration, $R_{\text {permission }}$ was set to 1 or to $R$ after the combination of relating three texture components. Permission ratio $R$ controls the orientation relation dependence of both boundary and triple line mobility. The effect of a boundary energy on the migration rate is also represented by $R$.

Thus, for simplification of the system, only two levels were considered for boundary energy and mobility of boundaries and triple lines, and a characteristic dependence on the orientation relations among associated grains was defined for the combination of texture components to which they belonged.

The coarsening process was executed by iteration of the systematic MC steps. ${ }^{5)}$ Non-zero energy cells were, at the beginning of a step, listed with their current energy.

In the main loop, for the sampling of passive cells, all cells in the list were, in descending order of the listed energy and randomly among the same energy, sampled once and only once from the list.

In the sub loop, for sampling of an active cell $j$ for the sampled passive cell $i$, cell $j$ was sampled from cells in the list and in the von Neumann neighborhood of cell $i$, in descending order of energy decrease (Eq. (2)) and randomly among the same energy decrease. Precedent orientation changes that occurred in the same MC step were taken into consideration to calculate Eq. (2). When the occupation of passive cell $i$ by active cell $j$ was permitted, the occupied cell was, in the same step, never again sampled as an active cell.

Primitive polycrystalline structures were created by random growth of seed cells. A specimen was divided into subspaces of $4 \times 4 \times 4$ cells. One of the cells in the $2 \times 2 \times 2$ center area of each subspace was selected at random as a seed cell. Seed cells were assigned systematic orientation numbers and grew similarly as in the case of the systematic MC steps, but only into their not-yet-defined neighboring cells. There were $256 \times 320 \times 256 / 64=327680$ grains in a primitive structure. Initial structures used for observation of characteristics of the grain coarsening process were then constructed via the grain coarsening process of the systematic MC steps with $R=1$ and $F=100$ until the number of grains decreased to approximately 7200 . The grains were homogeneously distributed with respect to the texture components.

\section{Basic Effects of $k T, R$, and $F$ on Boundary and Triple Line Migration}

Three grains were configured (Fig. 1) by stacking similar two-dimensional structures used for observation of triple point migration, ${ }^{5)}$ and the steady-state migration rate of triple lines was examined with $R=1$ and $F=100$ (Figs. 1(b) and 2). The number of protrusions on flat boundaries was counted at the same time (Figs. 1(c) and 2). On the basis of these experiments, parameter $k T$ was intuitively set to 0.87 , except in the case of Fig. 3 .

The dependence of the boundary migration rate on the permission ratio was investigated using the configuration in which two spherical grains were embedded in a matrix grain. One of the spherical grains shrunk with $R=1$, whereas the other spherical grain shrunk with $R \geq 1$. The shrinkage kinetics should be expressed by Eqs. (5) and (6), where parameter $K$ contains the product of boundary energy and boundary mobility and $V$ represents the volume of the shrinking grain:

$$
\begin{gathered}
d r / d t=-K / r \ldots \ldots \ldots . . \\
V^{2 / 3}=(4 \pi / 3)^{2 / 3}(-K t+C)
\end{gathered}
$$

The boundary energy (Eq. (1) with $f_{\text {in }}=100$ ) is independent of $R$; hence, $K$ should be proportional to the boundary mobility. Equation (6) holds for this simulation, as shown in Fig. 3(a). In the legend of Fig. 3(a), "systematic" indicates that the process proceeded under the systematic MC steps; in the case of "random," the sampling of passive cells in the main loop was executed irrespective of the listed energy and an active cell in a sub loop was sampled irrespective of an
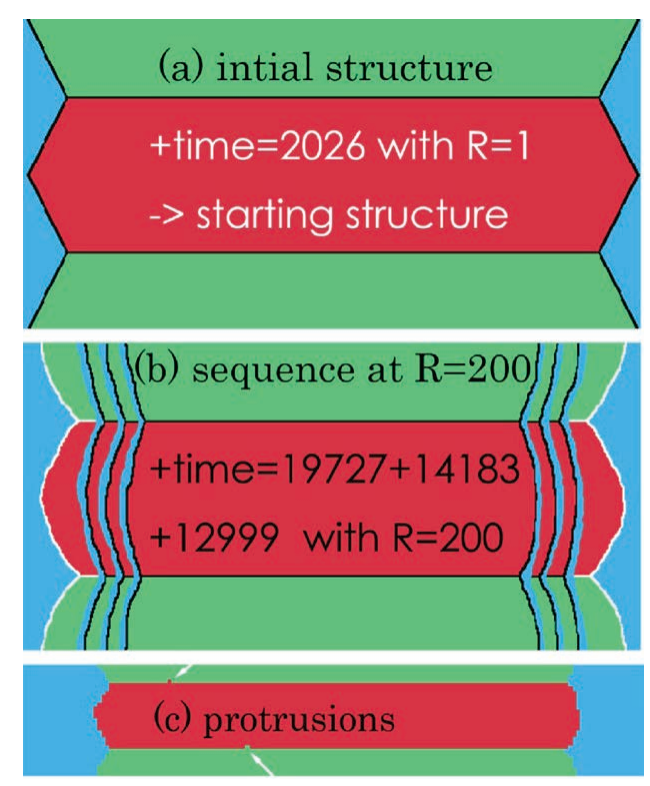

Fig. 1. Sections of three-grain specimens used to examine the triple line migration rate. Thickness of the centered red grain $a$ is equal to 140 in Figs. 1(a) and 1(b), and 32 in Figs. 1 (c) and 2. (Online version in color.)

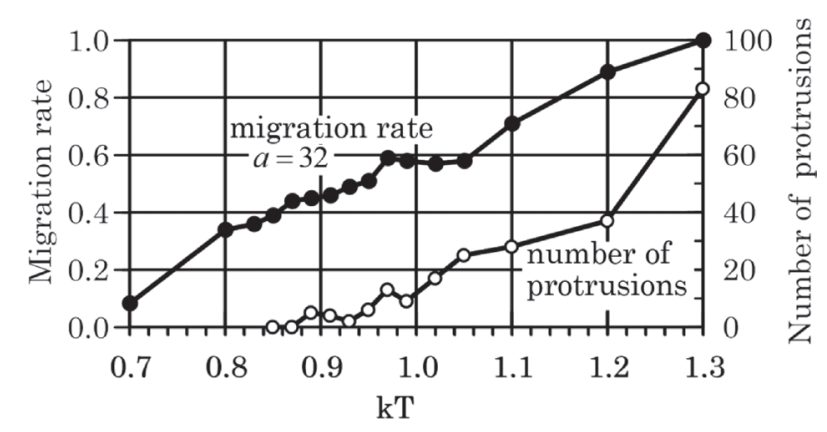

Fig. 2. Migration rate (relative) of a triple line and the number of protrusions (arbitrary units) on a flat boundary plotted against parameter $k T$. 

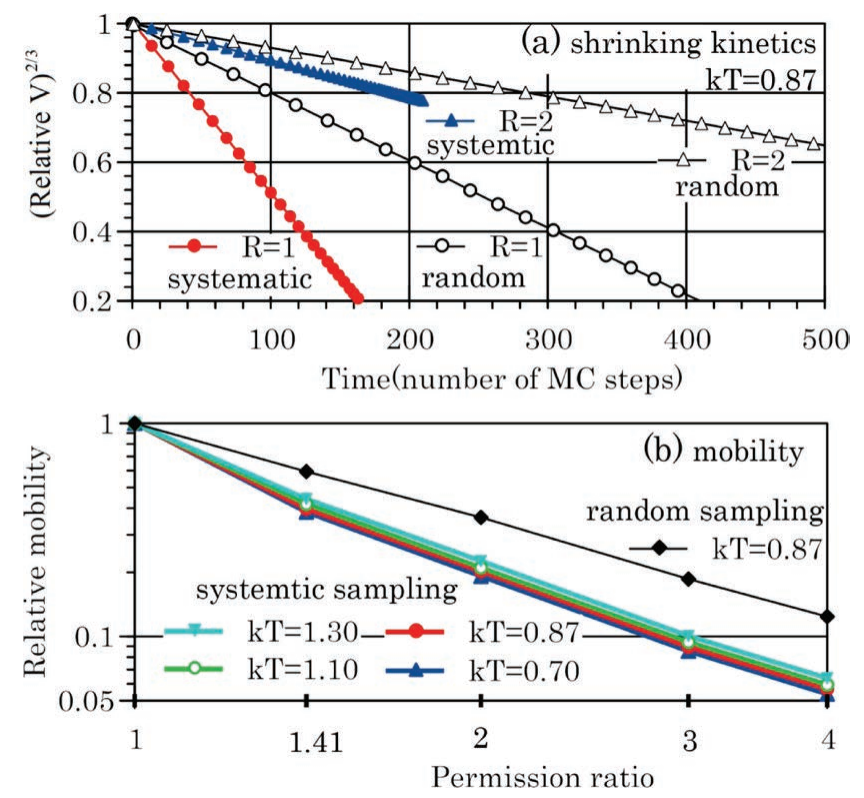

Fig. 3. Mobility of the boundary of shrinking spherical grains, as influenced by the permission ratio $R$. (Online version in color.)

energy decrease.

The relative boundary mobility obtained by observation of this configuration is shown in Fig. 3(b). The relative mobility depends only slightly on parameter $k T$. The dependence of mobility on parameter $R$ is certainly affected by the method of sampling in the MC step. ${ }^{6}$ ) The systematic sampling, however, indeed substantially reduces the computing time (Fig. 3(a)).

The boundary energy, Eq. (1), is defined quite locally. Figure 4 exemplifies the dependence of the average boundary energy on its orientation and the boundary energy factor. A cylinder was geometrically embedded in a cubic specimen. The surface energy between the cylinder and the matrix grain was set to zero. The axis of the cylinder was parallel to one of the principal axes of the array of cubic cells, and its length was equal to the edge length of the specimen. The cylinder was divided into two grains by a flat separating boundary that contained the principal axis of the cylinder, and the separating boundary was rotated step by step about the axis. At a rotation angle of zero, the boundary contained another principal axis of cubic cells. The energy, Eq. (1) with $f_{\text {in }}=F$, was, at each rotation angle, summed over the cells related to the separating boundary; the sum was then normalized with respect to the sum obtained for $F=100$ and a rotation angle of zero. The calculated area of the boundary to average its faceting structure - the radius of the cylinder that was sufficiently large to result in smooth curves - corresponded to 230 cells.

The dependence of the boundary migration rate on the boundary energy factor was similarly investigated using the configuration of two spherical grains in a matrix grain. In this case, $f_{i n}=F$ and $f_{i n}=100$ were respectively applied to the lower-boundary-energy grain and to the higher-boundary-energy grain. In Fig. 5, legend $p=p(\Delta E)$ indicates that $R_{\text {permission }}=1$ was substituted into Eq. (3) for both spherical grains, whereas legend $p=(\mathrm{F} / 100) \mathrm{p}(\Delta \mathrm{E})$ indicates that $R_{\text {permission }}=100 / F$ was applied to the lower-boundary-energy grain only. The axis of ordinates

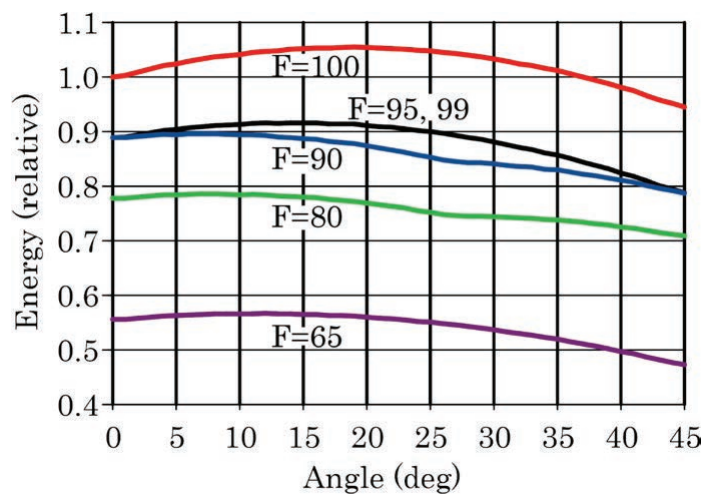

Fig. 4. Boundary energy calculated using the boundary energy factor $F$ and plotted against its orientation angle. (Online version in color.)
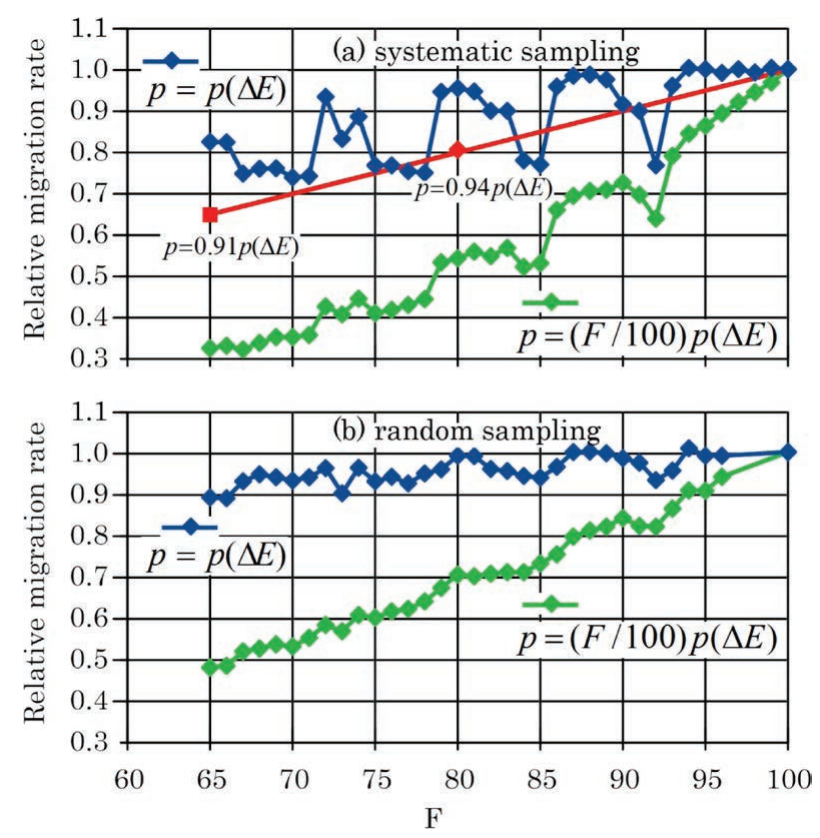

Fig. 5. Mobility of the boundary of shrinking spherical grains, as influenced by the boundary energy factor $F$ and the correlating permission ratio $R$. (Online version in color.)

in Fig. 5 corresponds to $K$ in Eq. (6). The term "relative" indicates that the migration rate was normalized with respect to the value obtained for the higher-boundary-energy grain.

Figures 5(a) and 5(b) corresponds to "systematic" and "random" sampling in the MC step, respectively. The curves in this figure show some singularity, which might be caused by expression of boundary energy (Eq. (1)) as an integer. In the case of simple $p=p(\Delta E)$, the dependence is mostly smaller than expected on the basis of the decrease in the boundary energy, or the decrease in $F$; by contrast, in the case of $p=(F / 100) p(\Delta E)$, the decrease in migration rate is larger than expected. When $p=0.94 p(\Delta E)$ and $p=$ $0.91 p(\Delta E)$ are respectively applied to the case of $F=80$ and $F=65$, the migration rates are proportional to factor $F$ (Fig. 5(a)).

We further examined the dependence of the boundary energy on factor $F$ by measuring equilibrium dihedral angles among three flat boundaries. A section of an initial configuration is shown in Fig. 6(a), and a three-dimensional specimen is constructed by its stacking. The configuration was not permitted to change outside of an inscribed cylin- 
der, whereas inside the cylinder, it was permitted to change so that the triple line migrated to attain equilibrium dihedral angles (Figs. 6(a) $\rightarrow$ 6(b) $\rightarrow$ 6(c)). In the initial configuration, the angle between the horizontal axis and the trace (that corresponded to the trace denoted by $\sigma 1$ in Fig. 7(a)) of one of the boundaries was set to $\alpha$ (in Fig. 6(a), $\alpha$ was $15^{\circ}$ ). Hereafter, $\sigma_{L}$ and $\sigma_{H}$ represent, respectively, the boundary energy calculated with $f_{\text {in }}=F$ and $f_{\text {in }}=100$. Notation HLL (Figs. 6(b), 6(c), 6(d)) indicates that boundary energy $\sigma$ l was $\sigma_{H}$, whereas boundary energies $\sigma 2$ and $\sigma 3$ were $\sigma_{L}$. Similarly, LHH (Fig. 6(e)) indicates that boundary energy $\sigma 1$ was $\sigma_{L}$, whereas boundary energies $\sigma 2$ and $\sigma 3$ were $\sigma_{H}$. When one of the three boundary energies $(\sigma 1, \sigma 2$, or $\sigma 3)$ is either $\sigma_{H}$ or $\sigma_{L}$ and the other two boundary energies are correspondingly $\sigma_{L}$ or $\sigma_{H}$, four ways exist to select $\sigma_{H}$ and $\sigma_{L}$ from the three boundaries. Energy ratio $\sigma_{L} / \sigma_{H}$ can be calculated two different ways from a set of three equilibrium dihedral angles obtained for each combination. Rotation angle $\alpha$ was varied from 0 to $45^{\circ}$ in $5^{\circ}$ steps. Therefore, 80 values of $\sigma_{L} / \sigma_{H}$ were observed (Fig. 8) for a given value of $F$ (ten levels for $\alpha$, four levels for a combination of higher and lower energy boundaries, and two levels for a combination of $\sigma_{L}$ and $\sigma_{H}$ ). Dihedral angles were experimentally calculated by the linear regression of coordinates of boundary traces in the mid layer (Fig. 7(b)). The regression was made for points located in a square area whose edges were 20 cells. In Fig. 8, legend "average" refers to the average of 80 values, and legend "boundary energy" refers to the relative boundary energy obtained by averaging (over rotation angles from 0 to $45^{\circ}$ ) along the curves shown in Fig. 4. The results in Fig. 8 indicate that, in the present model, the
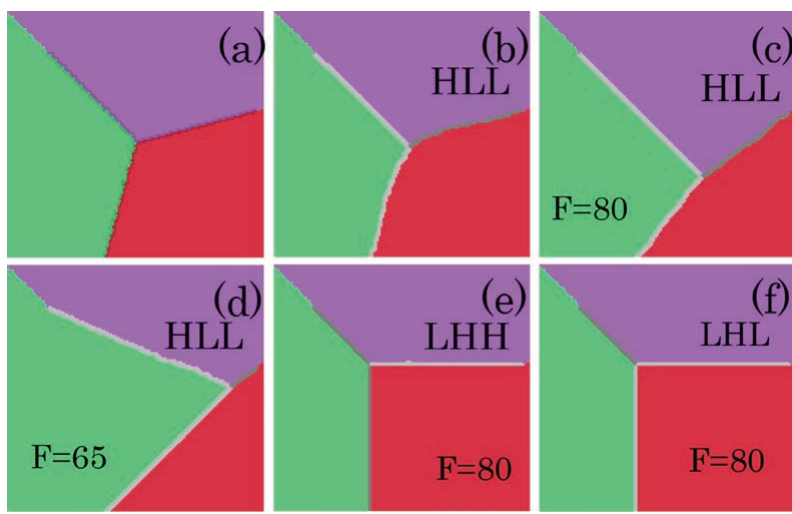

Fig. 6. Sections of three-grain specimens used to observe equilibrium dihedral angles. (Online version in color.)
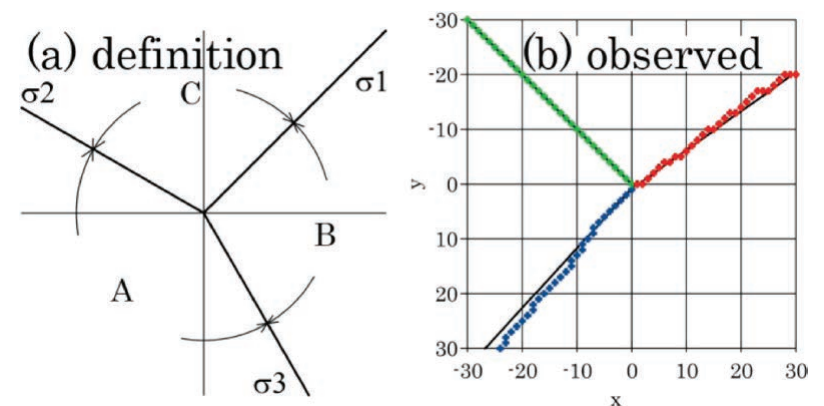

Fig. 7. Schematic presentation of dihedral angles. (Online version in color.) boundary energy certainly decreases with decreasing boundary energy factor. The large scatter of the values obtained by equilibrium dihedral angles must be due to the orientation dependence of boundary energy (Fig. 4).

The migration rate of triple lines was examined using the configuration shown in Fig. 1 . In this case, $f_{\text {in }}$ was 100 and $R_{\text {permission }}$ was set to 1 for boundary migration and to $R(\geq 1.0)$ for triple line migration. Equations (7)-(9) were theoretically obtained: ${ }^{5)}$

$$
\begin{gathered}
V(a)_{\infty}=2 m_{b} \sigma \arccos (\sin (\pi / 3)) / a \ldots \ldots \ldots \\
V(a) / V(a)_{\infty}=\arccos (\sin \theta) / \arccos (\sin (\pi / 3)) \\
\Lambda=a m_{t l} / m_{b}=2 \arccos (\sin \theta) /(1-2 \cos \theta) \ldots
\end{gathered}
$$

Here, $\sigma$ denotes boundary energy, $V(a)$ represents the triple line migration rate as a function of the thickness of shrinking grain $a, \theta$ is the dihedral angle of the migrating boundary, and $m_{t l}$ and $m_{b}$ represent the mobility of the triple line and the mobility of the boundary, respectively. When $m_{t} / m_{b}$ approaches $\infty$, or when the triple line does not drag on a boundary, $\theta$ and $V(a)$ approach $\pi / 3$ and $V(a)_{\infty}$, respectively. The theoretical curve ("theory" in Fig. 9) was constructed on the basis of Eqs. (8) and (9). In the case of experimentally observed data, $V(a)_{R} / V(a)_{\mathrm{R}=1}$ corresponding to $V(a) / V(a)_{\infty}$ was plotted against $\Lambda$ calculated using Eq. (10):

$$
\Lambda=a M /(R-1) \text {. }
$$

Parameter $M(=0.965)$ is used to minimize the devia-

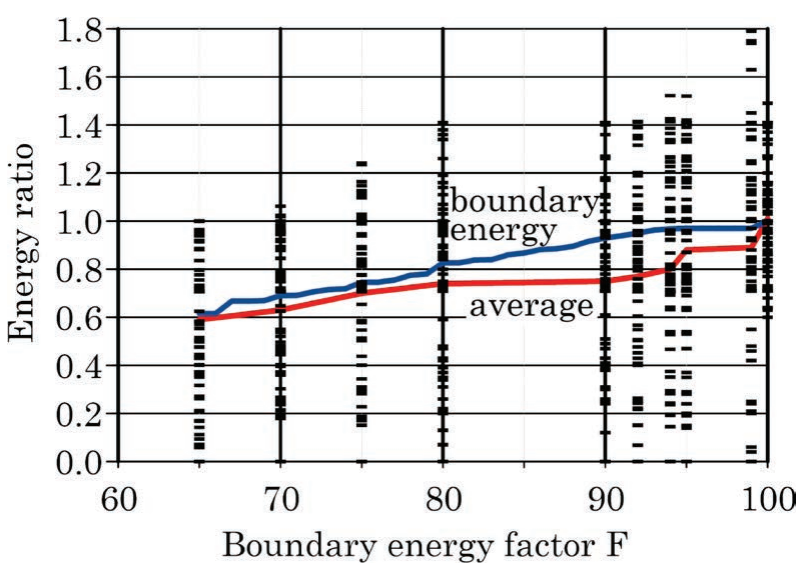

Fig. 8. Boundary energy determined from equilibrium dihedral angles plotted against the boundary energy factor $F$. (Online version in color.)

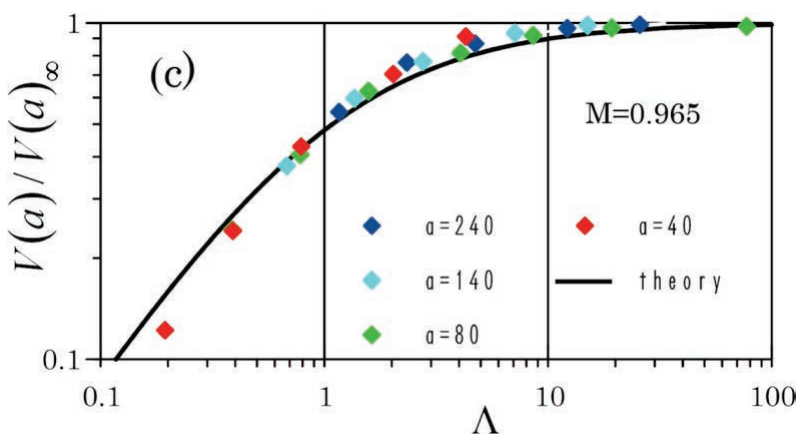

Fig. 9. Migration rate of a triple line, as influenced by the permission ratio $R$. (Online version in color.) 
tion of experimental data from the theoretical line. The deviation must arise from the orientation dependence of energy and the mobility of the boundary and triple line. These notations are essentially the same as those used in the two-dimensional case. ${ }^{5}$

\section{Characteristics of the Grain Coarsening Process Observed in the Present Model}

Grain structures obtained by the fundamental grain coarsening process, namely results for structures observed with $f_{\text {in }}=100$ and $R_{\text {permission }}=1$ are presented in this section. Effects of orientation-relation-dependent $f_{\text {in }}$ and $R_{\text {permission }}$ on the grain growth texture development shall be demonstrated in the following report.

The coarsening ratio was defined as the "number of grains in an initial structure" divided by "number of grains after coarsening." The ordinate axis in Fig. 10 corresponds to the number of grains that exhibited the specified number of faces. "Fraction" refers to the number of grains divided by the total number of grains in a specimen. The grain size distribution shows, at least within an observed range, a systematic change with increasing coarsening ratio.

The histograms in Fig. 10 (they will be named in this report as "topological grainsize distribution") are within the fluctuation reported using Surface Evolver, ${ }^{9)}$ phasefield, ${ }^{10,11)}$ front-tracking, ${ }^{12)}$ and $\mathrm{MC}^{13)}$ simulations. Pure iron grains showed a slightly deviated distribution, whereas the distribution of titanium grains coincided better with the simulated distribution. ${ }^{13)}$ Small changes in the distribution curves with progression of coarsening have also been reported. ${ }^{11)}$ The authors of previous papers plotted the fraction on a linear scale, which may have led them to not notice the small but systematic changes in the peripheral regions. A curve derived by Mullins ${ }^{14)}$ on the basis of his model was slightly different; however, Rios and Glicksman ${ }^{15}$ could adjust their model to a simulated one.

The normalized volume was defined as the ratio between the volume of a grain, $V$, and the average volume of grains in a specimen, $\bar{V}$. The normalized volume-equivalent radius, $R / \bar{R}$, is equal to $(V / \bar{V})^{1 / 3}$. Figure 11 shows its dependence on the number of faces. A ratio was calculated for a mean of grains with a specified number of faces. The legends are the same as in Fig. 10. The correlation between volumeequivalent radius and the square root of the number of faces is not a straight line but rather a sigmoidal curve, as was the case in a phase-field simulation. ${ }^{11)}$ The observed correlation is very similar to that reported for a phase-field simulation ${ }^{11)}$ but differs slightly from the correlations deduced from Evolver $^{9)}$ and $\mathrm{MC}^{7)}$ simulations.

The Aboav-Weaire ${ }^{16)}$ plot observed in the structure with a coarsening ratio of 4 is shown in Fig. 12. In the legends, $N$ represents the number of faces, and suffix $i$ denotes the serial number of neighboring grains. Plotted data were calculated as explained for Fig. 11. An equation obtained by linear regression $\left(\sum N_{i}=13.58 N+25.6\right)$ approximately agrees with the reported equations. ${ }^{9,10)}$ Statistically, for a grain with $N=15.26$, the mean number of faces of its neighboring grains $\sum_{i} N_{i} / N$ is equal to $15.26 ; N=15.26$ represents, in

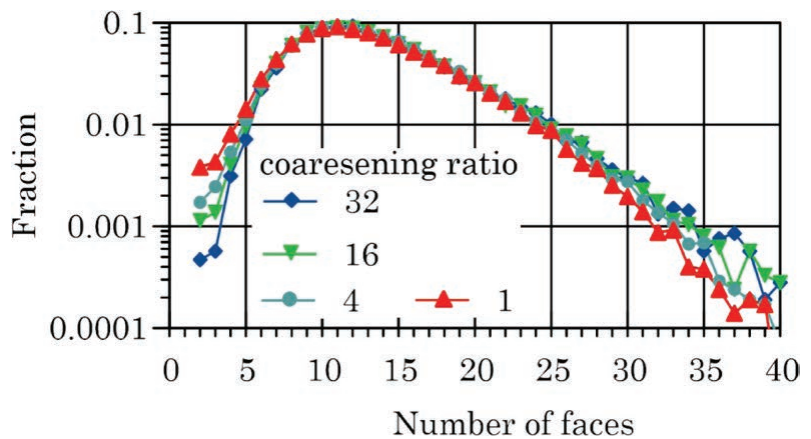

Fig. 10. Topological grain size distribution, as influenced by the coarsening ratio. (Online version in color.)

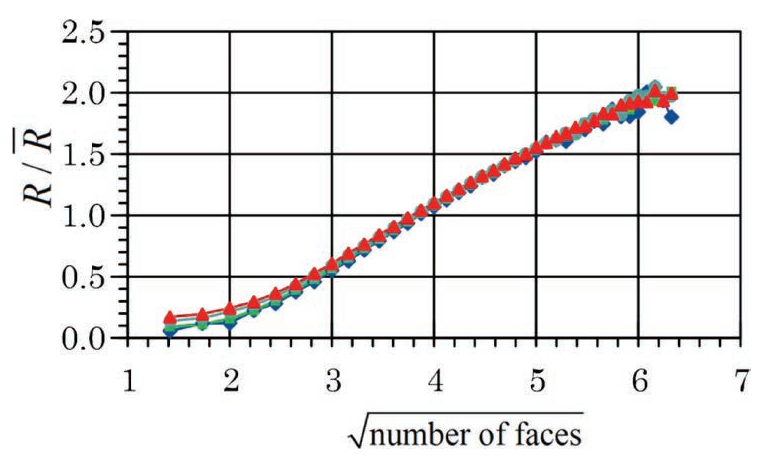

Fig. 11. Statistical correlation between volume-equivalent radius and the number of faces of a grain. (Online version in color.)

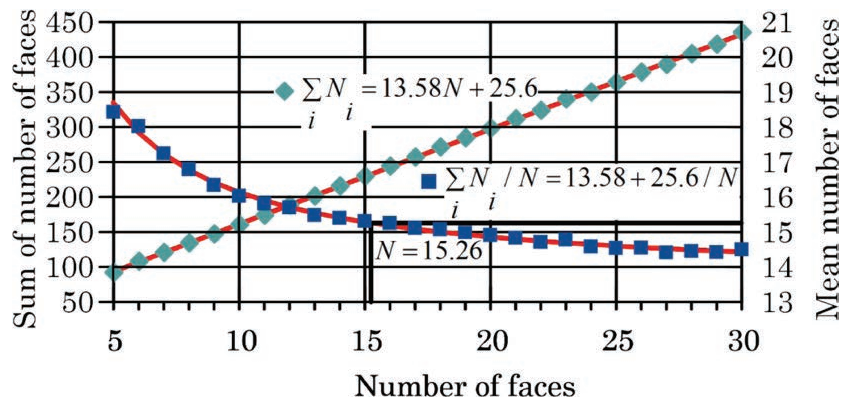

Fig. 12. The Aboav-Weaire law in the present model. (Online version in color.)

this sense, the topological mean grain size of this structure. It corresponds to a normalized volume of 1.13 (see Fig. 11).

Figure 13 shows the volume change of grains at a stage of coarsening ratio $=4$ after one systemic MC step, where the number of grains in a specimen decreased by $1.0 \%$. Here $V$ represents the mean volume of grains classified by the number of faces (Fig. 13(a)) or normalized volume (Fig. 13(b)). The figures demonstrate that the critical grain size to grow or shrink in a short period corresponds to the topological mean size.

The critical number of faces of a grain $N_{c}$ determined by Evolver, ${ }^{9)}$ phase-field, ${ }^{11)}$ and $\mathrm{MC}^{17)}$ methods agree with the present value of approximately 15 and indicate that the critical equivalent radius is larger than the mean equivalent radius. ${ }^{11,17)}$ Mean field calculations, ${ }^{14,15,18)}$ however, indicated mostly smaller $N_{c}$ values of approximately 13, although the most recently reported calculation ${ }^{19)}$ shows that $N_{c}$ is nearly 15 . In any event, the author's opinion is that the 

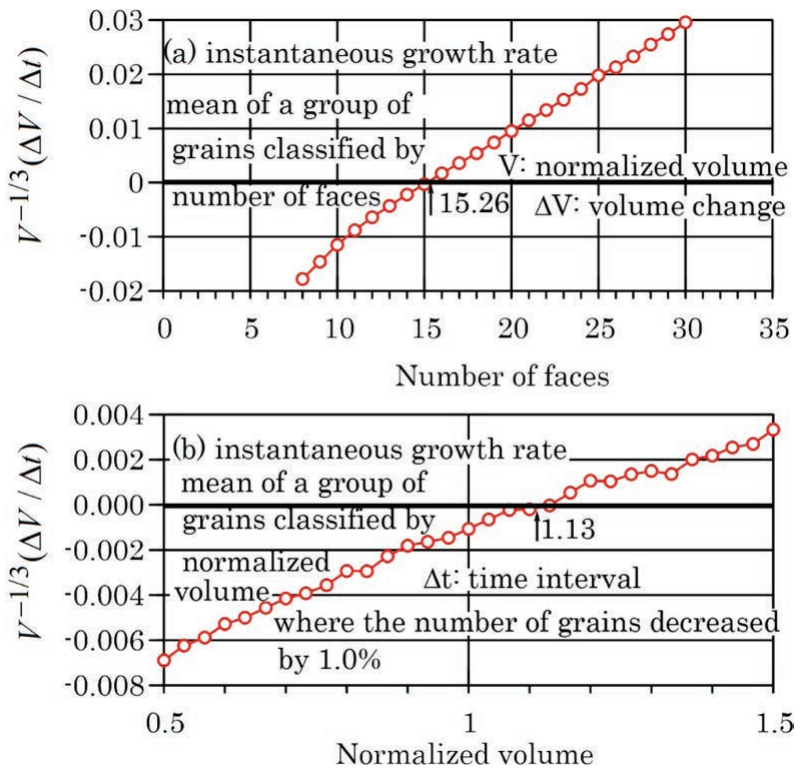

Fig. 13. Change in the mean volume of classified grains in a single systematical MC step. (Online version in color.)

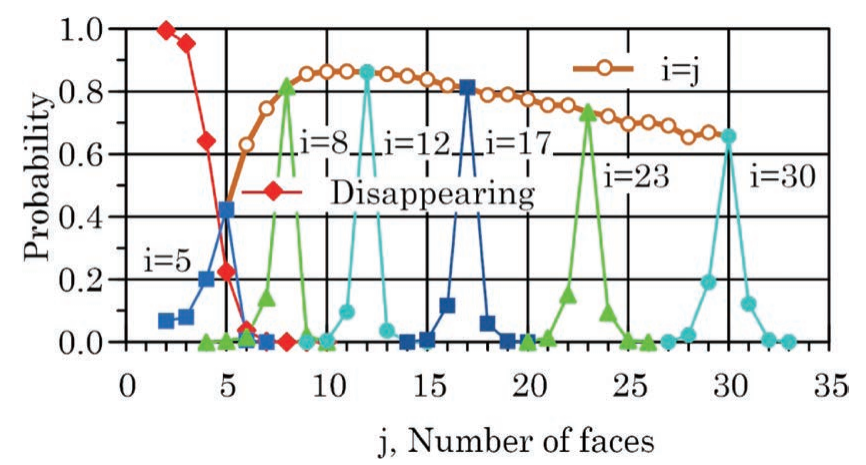

Fig. 14. Transition probability of the number of faces of a grain from $i$ to $j$ in a single systematical MC step.

critical size corresponds to the topological mean grain size.

Figure 14 shows three probabilities as a function of the number of faces observed at the same stage as that represented in Fig. 13. The curve labeled "Disappearing" represents the probability with which grains disappear in the short period. The curve labeled " $i=j$ " shows the probability with which the number of faces of grains remains unchanged. Curves labeled " $i=5, "$, $i=8$," and so on demonstrate the changing probability of the number of faces of grains from $i$ to $j$. The results in this figure indicate that some grains smaller than the topological mean are likely to exhibit an increase in their number of faces within a short period, whereas the number of faces of a grain never increases on average but absolutely decreases, even if the size of a grain is far larger than the topological mean. These probabilities as a function of topology class have not yet been reported.
They support the opinion that the topological space is an important consideration in the grain coarsening process. ${ }^{11)}$

\section{Summary}

Details of a modified Potts-MC-type grain coarsening three-dimensional model that effectively reduces computing time were explained. Effects of parameters in the model to control anti-freezing of the structure, orientation-relation-dependent migration rate and energy of boundaries as well as orientation-relation-dependent migration rate of triple lines on the elementary structural development process were demonstrated.

The topological grain size distribution, Aboav-Weaire plot, and critical sizes of grains necessary to grow or shrink within a short time, was observed by the model and approximately coincided with the results of previously reported simulations and with the results of mean field model calculations. The present modelling can be said to represent essential features of the grain coarsening process.

The critical grain size was found to coincide with the topological mean grain size. The number of faces of grains of this size is, according to the Aboav-Weaire law, equal to the mean number of faces of its neighboring grains.

The transition probabilities of grains between topological classes in the shortest elapsed time were observed for the first time. A topological class of any grain, regardless of whether its current size is larger than the mean, continues, on average, to decrease, and any grain finally disappears. These observations suggest that an approximate steadystate topological grain size distribution could exist for only a short period.

\section{REFERENCES}

1) F. J. Humphreys and M. Hatherly: Recrystallization and Related Phenomena, 2nd ed., Pergamon Press, Oxford, (2004).

2) A. D. Rollett: JOM, 56 (2004), Issue 4, 63.

3) A. Brahme, J. Fridy, H. Weiland and A. D. Rollett: Model. Simul. Mater. Sci. Eng., 17 (2009), No. 1, 015005.

4) K. Ito: IOP Conf. Ser.: Mater. Sci. Eng., 82 (2015), 012043.

5) K. Ito: Comput. Mater. Sci., 62 (2012), 117.

6) E. A. Holm, M. A. Miodownik and A. D. Rollet: Acta Mater., 51 (2003), 2701.

7) D. Zoellner: Comput. Mater. Sci., 118 (2016), 325.

8) J. K. Mason, J. Lind, S. F. Li, B. W. Reed and M. Kumar: Acta Mater., 82 (2015), 155.

9) F. Wakai, N. Enomoto and H. Ogawa: Acta Mater., 48 (2000), 1297.

10) Y. Suwa, Y. Saito and H. Onodera: Comput. Mater. Sci., 40 (2007), 40.

11) R. D. Kamachali and I. Steinbach: Acta Mater., 60 (2012), 2719.

12) E. A. Lazar, J. K. Mason, R. D. MacPherson and D. J. Srolovitz: Acta Mater., 59 (2011), 6837.

13) W. Xue, H. Wang, G. Liu, L. Meng, G. Ma and M. Feng: Mater. Lett., 174 (2016), 171.

14) W. W. Mullins: Acta Metall., 37 (1989), 2979.

15) P. R. Rios and M. E. Glicksman: Acta Mater., 56 (2008), 1165.

16) S. F. Edwards and K. D. Pithia: Phys. A, 205 (1994), 577.

17) D. Zoellner and P. Streitenberger: Scr. Mater., 54 (2006), 1697.

18) S. Hilgenfeldt, A. M. Kraynik, S. A. Koehler and H. A. Stone: Phys. Rev. Lett., 86 (2001), 2685.

19) R. T. DeHoff, B. R. Patterson, C. A. Sahi and S. Chiu: Acta Mater., 100 (2015), 240. 